\title{
Treatment outcomes of the posterolateral approach of plate fixation for posterior pilon fractures
}

\author{
MAOFENG GAO, NAICHENG LIU, YU CHENG, WEIDONG SHI and HUILIN YANG \\ Department of Orthopaedics, The First Affiliated Hospital of Soochow University, \\ Suzhou, Jiangsu 215000, P.R. China
}

Received September 27, 2018; Accepted February 21, 2019

DOI: $10.3892 / \mathrm{etm} .2019 .7458$

\begin{abstract}
Posterior pilon fracture is a common type of intraarticular fracture encountered in clinical practice. The treatment of this fracture pattern has been increasingly reported. However, methods for minimizing the associated surgical trauma and achieve effective fixation still require to be established. The present study involved 23 patients with posterior pilon fracture treated at the First Affiliated Hospital of Soochow University (Suzhou, China) between March 2013 and October 2017. Klammer's classification system was used to divide the posterior pilon fractures into 3 types. The surgical procedure, reduction of post-operative fracture, peri-operative complications and post-operative functional recovery were reviewed and analyzed. The reduction in post-operative fractures was evaluated by determining the Burwell-Charnley scores at the last follow-up. Anatomical reduction was confirmed in 17 patients, and an acceptable reduction was reported in 6 patients. The American Orthopedic Foot and Ankle Score was used to assess ankle function recovery; the average score was 82.3 points (range, 44-97 points). In conclusion, the posterolateral approach is able to achieve anatomical exposure of the operative field. In addition, the posterior fracture fragment of the tibia may be fully exposed through the fibula fracture gap by retraction. Posterior placement of the plate may serve a definitive role in the fixation of the posterior fracture fragment and maintain stability in the anatomical reduction of the fracture, which is helpful in early functional rehabilitation.
\end{abstract}

\section{Introduction}

Posterior pilon fracture is a specific type of fracture that manifests in a similar manner to either an ankle fracture or a pilon fracture. Posterior pilon fractures are caused by rotational or

Correspondence to: Dr Huilin Yang, Department of Orthopaedics, The First Affiliated Hospital of Soochow University, 188 Shizi Road, Suzhou, Jiangsu 215000, P.R. China

E-mail: 228793567@qq.com

Key words: posterior pilon fracture, internal fixation, fracture fixation axial forces and lead to posterior distal tibial fractures with displacement toward the proximal direction. Posterior pilon fractures are also frequently accompanied by talus dislocation $(1,2)$. Notably, posterior articular fractures of the distal tibia may be divided into posterior malleolus fractures and posterior pilon fractures. Avulsion fractures caused by torsion trauma are types of posterior malleolus fractures, which are characterized by small fracture blocks and do not involve the joint surface or a small part of the joint surface. If the injury is associated with vertical trauma, it is a pilon fracture, and the fracture fragment is generally large, involving the articular surface and is displaced proximally (3). In clinical practice, posterior pilon fractures are not uncommon. Post-operative articular function is poor due to difficult reduction processes and complex ankle ligament injury; post-operative traumatic arthritis, even failure of internal fixation and fracture re-displacement, may occur $(3,4)$.

Regardless of the type of articular fracture, anatomic reduction, reliable fixation and early functional exercise are considered the basis of surgical treatment. Posterior tibial fracture fragments, incarcerated tissue and die-punch fragments are common (5). The reduction procedure requires clear visualization of the surgical field and minimal operative trauma. Therefore, it is required to select the appropriate incision and approach. In addition, exposing the fragments posterior to the tibia and the incarcerated tissue between the bone fragments are key to a successful surgery (5). In terms of selecting a fixation technique, cannulated compression screws (from anterior to posterior, and vice versa) and posterior tibia plates are commonly used for fixation $(1,2,5)$. Compared with compression screws, fixation with a posterior tibia plate is more secure and stable, and helps in the early functional rehabilitation of the ankle $(2,6)$. The aim of the present study was to retrospectively review the procedure and determine prognostic factors in patients with posterior pilon fracture diagnosed at our hospital to provide recommendations for its clinical treatment.

\section{Patients and methods}

Patient cohort. A total of 23 patients with posterior pilon fractures were recruited at the First Affiliated Hospital of Soochow University (Suzhou, China) between March 2013 and October 2017. The cohort comprised 9 males and 14 females with a mean age of 46.7 years (range, 19-72 years). 
The causes of injury included traffic accidents $(n=11)$, falls when walking $(n=6)$ and falls from a high place $(n=6)$. A total of 4 cases were accompanied by rib fractures and lung contusion, 2 with open injuries, 2 with pelvic fractures and 1 with traumatic subarachnoid hemorrhage. Pre-operative computed tomography (CT) plain scanning, coronal and sagittal image reconstruction and articular surface image reconstruction were performed in all patients. Klammer et al classification system (1) was used to divide posterior pilon fractures according to the shape of the fracture line on the axial image into type I (5 patients), type II (10 patients) and type III (8 patients) fractures. According to the AO classification (7) of ankle fractures, the patients in this group included 5 cases of the B3 type, 4 cases of the $\mathrm{C} 1$ type, 6 cases of the $\mathrm{C} 2$ type and 7 cases of the $\mathrm{C} 3$ type. Following admission, the patients were evaluated based on the swelling of the ankle. Of these patients, 4 underwent emergency surgery within $8 \mathrm{~h}$ post-injury, 13 underwent cast immobilization of the ankle in a neutral position, and 6 underwent calcaneal traction and then received surgical treatment when the ankle swelling subsided and ankle skin striae appeared. In the patients who underwent non-emergency surgery, the average pre-operative duration was 8.5 days (range, 5-24 days).

Surgical technique. General anesthesia via inhalation and intravenous routes was used for surgery. The patients were placed in either the prone or the prone float position with elevation of the affected lower extremity with a slightly bent knee. This position allowed for the extreme extension of the ankle during the reduction procedure. A longitudinal incision was made between the lateral margin of the Achilles tendon and the posterior margin of the fibula. The distal incision was curved forward and the incision was free for extension in either direction as required (Fig. 1). The sural nerve and the small saphenous vein were identified, exposed and protected by skin flap traction (Fig. 2). The posterior and lateral fibula was visualized via the anterior space of the peroneus longus and brevis muscles. At the site posterior to the peroneus longus and brevis muscles, the muscle belly of the flexor hallucis longus muscle was separated by blunt dissection between the interosseous membrane and the lateral tibia. Care was taken to protect the peroneal artery when operating through this access. The flexor hallucis longus muscle was retracted medially to expose the posterior tibial fracture fragments. In cases with incarcerated soft tissue and die-punch fragments between the fracture fragments, Kirschner wires were placed on either side of the fracture line; a Kirschner wire retractor was then used to retract the Kirschner wires to allow for the separation of the fractured bone. At this point, it was possible to fully visualize the fibular fracture fragments as well as the incarcerated soft tissue and bone fragments in the fracture gap (Fig. 3). A small curette was used to remove the incarcerated tissue from the tibial and fibular fracture gaps. First, the fractured fibula was reduced via temporary Kirschner wire fixation. The fracture fragment posterior to the tibia was exposed. The fracture fragment could not be turned over due to the limited exposure of the posterior tibia and the intact inferior tibiofibular ligament during all the surgical procedures; however, the incarcerated tissue between the fragments was removed via the fibular fracture gap. This posterior fragment was then slightly elevated to separate it from the tibia. The ankle was then subjected to extreme extension to facilitate complete anatomical reduction. A Kirschner wire was then used to temporarily fix the posterior fragment of the ankle. Intra-operative fluoroscopy was performed to assess whether the articular surface was smooth (Figs. 4 and 5). A posterior-lateral plate and screws were used to fix the fibula. A specific anatomic locked plate or a pre-bent distal radius plate was used to fix the posterior fragments of the tibia. According to the conditions of the medial malleolus fracture, a posterior-medial or medial incision was made for medial malleolus fracture reduction and fixation. The tourniquet was then released and adequate hemostasis was achieved; subsequently, the incision was closed in layers. Drainage of the incision was not required and the incision was dressed with sterile dressings. The ankle was fixed via cast immobilization in a neutral position. A total of 15 patients had die-punch fragments between the tibia fracture fragments, and 10 patients received posterior-medial or medial incisions for medial malleolus fracture reduction and fixation; this combination was utilized as the medial malleolus fracture and the fracture involving the posterior malleolus colliculus require the posterior medial incision for reduction. It was difficult to expose the posterior fracture fragment of the tibia via the fibula fracture gap when the fracture lines of the tibia and fibula were not at the same height. In such cases, it was necessary to extend the incision and expose the fracture from behind the fibula. Images of typical cases are presented in Figs. 6-8.

Post-operative treatment and follow-up. Based on the fracture conditions, patients started non-weight-bearing functional exercise on the second day post-surgery if no ligament damage was present. Otherwise, the patients were placed in a cast boot or a splint for 3 weeks and started performing active range of motion exercises. The patients were allowed full weight bearing following 6 weeks. Imaging evaluation was performed using the Burwell-Charnley scoring system, which focused on the degree of displacement of the medial and lateral malleolus (8). Fractures were considered clinically healed when the patient was able to bear proper weight and experience no pain in the affected extremity and no pain upon palpation/percussion. The mean American Orthopedic Foot and Ankle Score (AOFAS) was 82.3 points (range, $44-97$ points) at the final follow-up visit.

\section{Results}

Preoperative treatment. The mean follow-up period was 14.5 months (range, 6-24 months). Following surgery, 17 patients were placed in a cast boot for immobilization. 6 patients were free from post-operative immobilization and allowed to start early motion exercises of the ankle. The mean fracture recovery time was 2.1 months (range, 2-3 months). The mean time to full weight-bearing walking was 2.4 months.

Clinical and radiological outcomes. Post-operative fracture reductions were evaluated using Burwell-Charnley scores. Anatomical reduction was confirmed in 17 patients $(73.9 \%)$ and an acceptable reduction was observed in 6 patients (26\%). At the final follow-up visit, the mean AOFAS was 82.3 points (range, 44-97 points). Good AOFASs ( $>75$ points) were reported in 20 patients, accounting for $86.9 \%$ of all patients. 


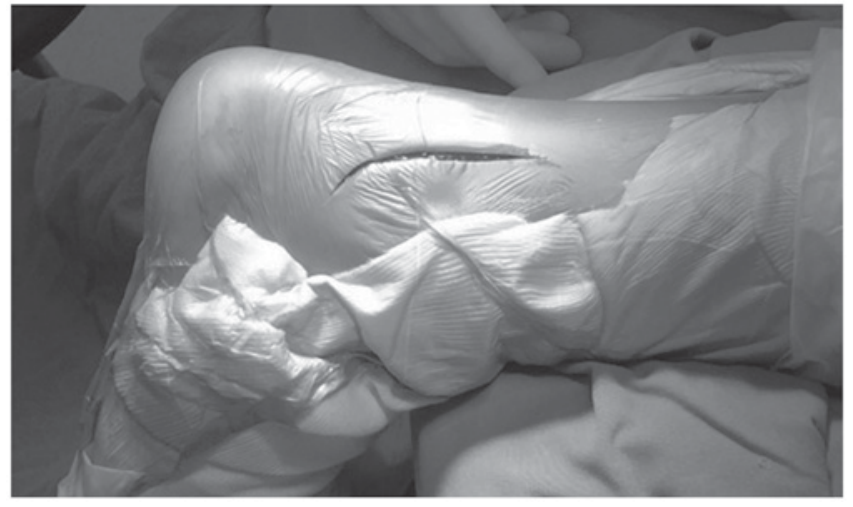

Figure 1. A skin incision was made between the lateral edge of the Achilles tendon and the posterior edge of the fibula.

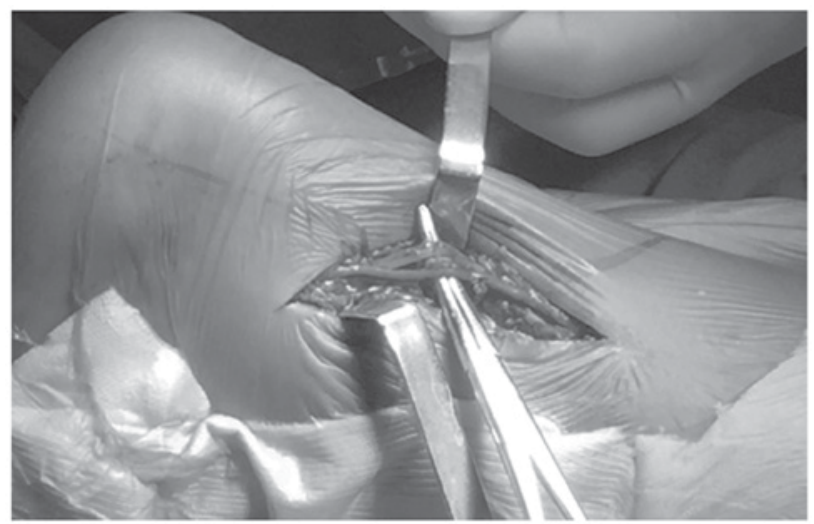

Figure 2. The sural nerve and small saphenous vein were identified and protected with a skin graft.

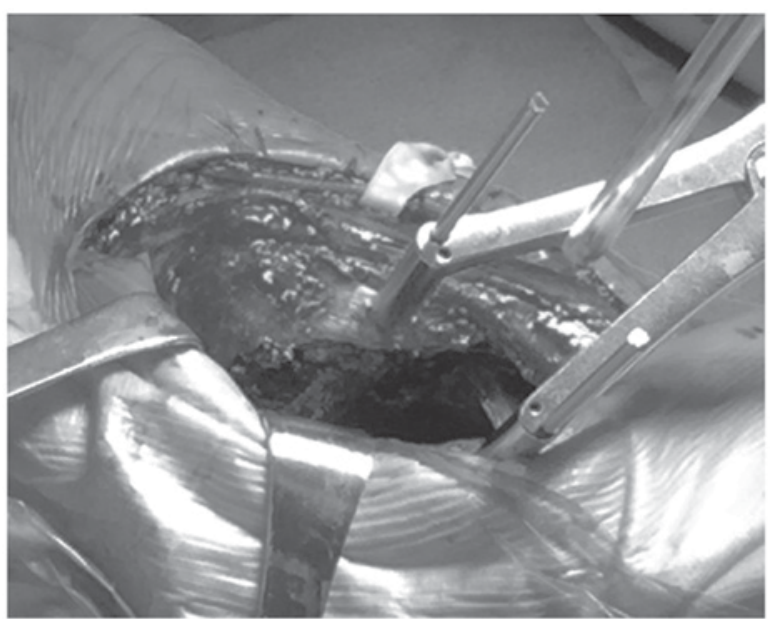

Figure 3. Fibula fracture fragments were retracted to gain access to the posterior fragment of the tibia. The soft tissue and the die-punch fragments incarcerated between the fragments were removed.

Complications. Postoperative complications occurred in 5 patients $(21.7 \%)$. One patient had a post-operative wound infection (no osteomyelitis and no plate exposure), and the wound healed following localized treatment. One patient had post-operative numbness around the incision and the dorsum of the foot that was caused by intra-operative nerve retraction;

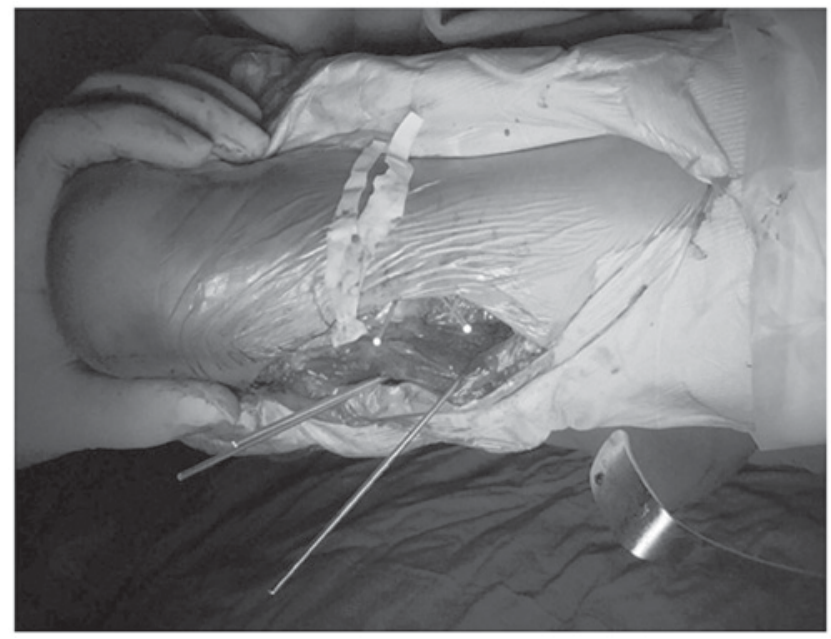

Figure 4. Exposure of the posterior tibial fracture line and reduction of the fractured fragments.

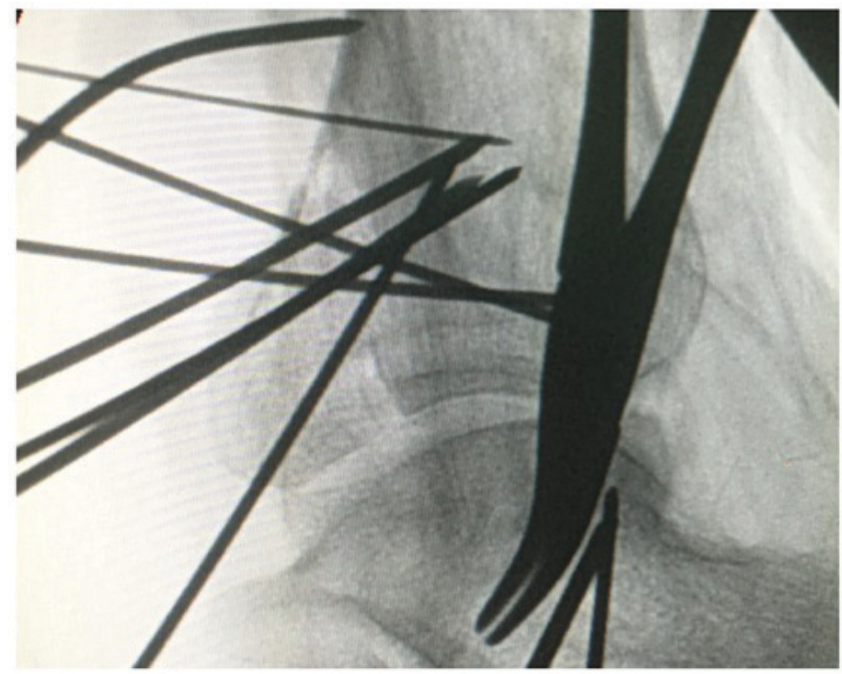

Figure 5. Intra-operative X-ray fluoroscopy presenting a satisfactory reduction and a smooth articular surface.

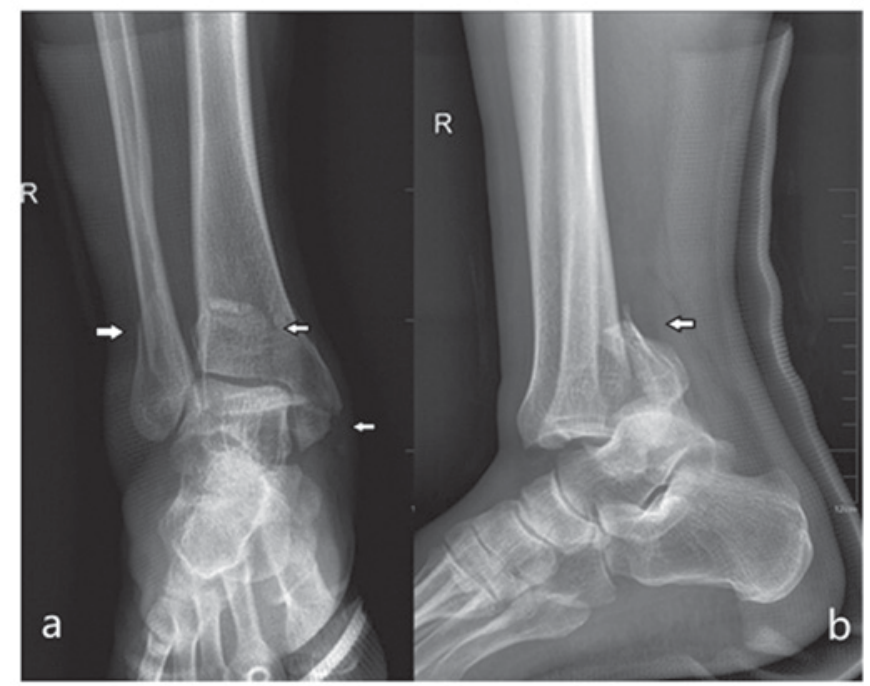

Figure 6. A 50-year-old male was diagnosed with posterior pilon fracture. Fractures of the posterior malleolus can be identified by the (a) anteroposterior and (b) lateral radiographs (arrows indicate the fracture lines). 


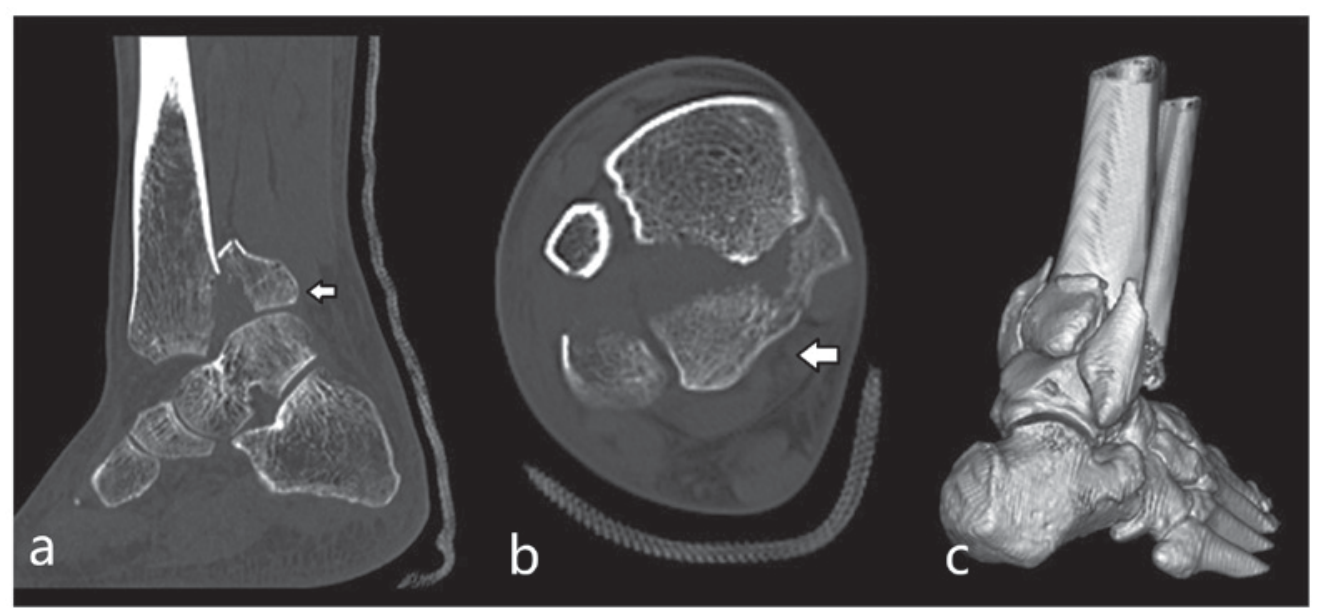

Figure 7. (a) Computed tomography imaging reconstruction clearly revealed the shape of the fracture fragments and the displacement direction. (b) On axial view, the Walkman bone fragments were visible. (c) CT surface reconstruction idnicated the morphology of the fracture. The arrows indicate the fracture fragments.

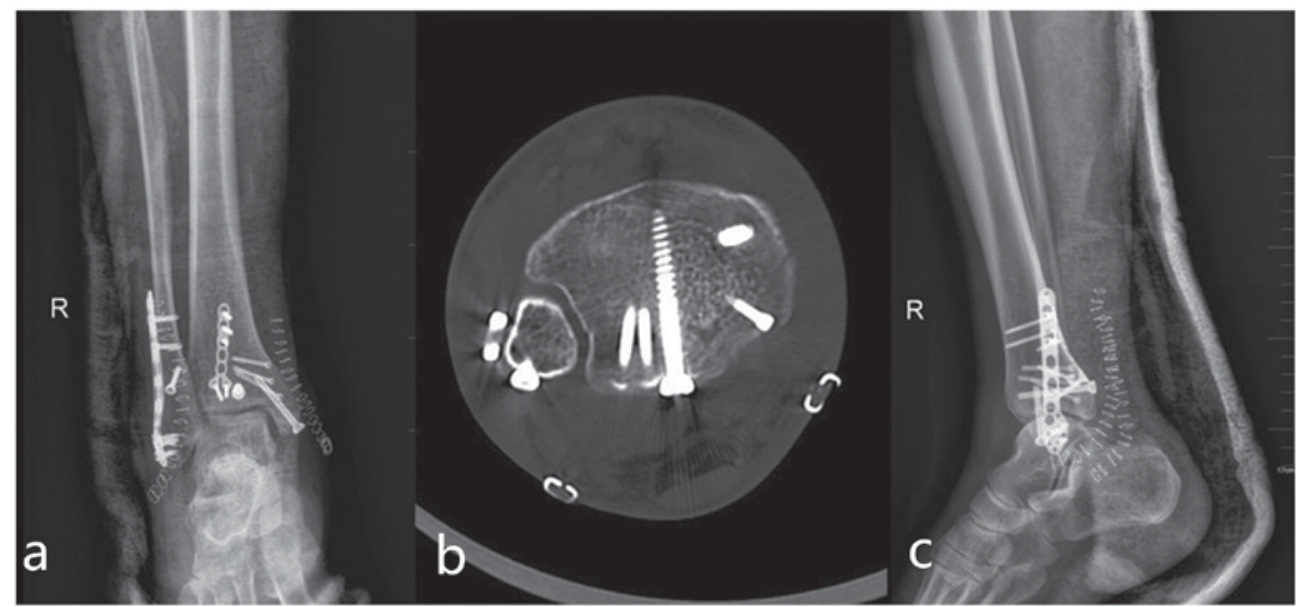

Figure 8. (a) Postoperative anteroposterior radiograph revealed fracture reduction and internal fixation. (b) Postoperative axial computed tomography scan indicated anatomical reduction of the syndesmosis. The screws were substantially perpendicular to the fracture line. The pre-bending orthopedic plate posterior to the tibia fitted the contour of the tibia. (c) Postoperative lateral radiography indicated that the ankle joint surface was smooth.

these symptoms gradually subsided 4 months following treatment with a neurotrophic agent. 3 patients complained of persistent pain and discomfort following surgery even though their fractures had been clinically healed. Their internal fixations were removed at 10,13 or 14 months post-surgery, and they recovered well following the removal of the internal fixation.

\section{Discussion}

Posterior pilon fractures are a specific type of intra-articular fracture in the distal tibia and are caused by low-energy rotational forces (posterior ankle fractures) or high-energy axial forces (pilon fractures). Amorosa et al (2) summarized the characteristics of posterior pilon fractures as follows: i) The posterior fracture fragment is large; ii) multiple fracture fragments may be present; iii) a 'stair' phenomenon is observed; and iv) fracture fragments are displaced toward the proximal direction. In clinical practice, posterior pilon fractures are not uncommon. Switaj et al (3) revealed that posterior pilon fractures accounted for $20 \%$ of fractures in a series of 270 patients with ankle fractures who underwent surgical treatment.
Topliss et al (4) analyzed the fracture lines of the distal tibia on axial views of CT images; posterior pilon fractures, presenting with coronal fracture lines and longitudinal displacement, accounted for $5.6 \%$ of all types of fracture. Forberger et al (5) performed a retrospective analysis of 45 patients with ankle fractures and reported that proximal displacement of the posterior fracture fragment was present in $73 \%$ of all ankle fractures. These results indicated that longitudinal forces were involved the fractures, and that they may be classified as posterior fractures. As displacement of the posterior fragment in the distal tibia is usually accompanied by posterior subluxation of the talus and posterior ligament complex damage, this type of fracture has a poor prognosis, with high probabilities of internal fixation failure and traumatic arthritis. In the literature, this particular type of fracture has drawn increasing attention $(3,4)$.

Multiple classifications of posterior pilon fracture have been used. Based on the location of the fracture line, Wang et al (6) divided posterior pilon fractures into two types. In type I fractures, two separated posterior fracture fragments, a posterior-medial fracture fragment and a medial malleolus fracture fragment are present, with the fracture line located above the lower 
syndesmosis. In type II fractures, the posterior-medial fracture fragment is adjacent to the medial malleolus fracture fragment; the fragments are displaced proximally, small incarcerated bones may be present and the fracture line is located beneath the lower syndesmosis. Klammer et al (1) retrospectively analyzed patients with posterior pilon fracture and divided those fractures into 3 types according to their increasing degree of complexity. In type I fractures, a single posterior malleolar fragment is present, and a long oblique fracture line runs through the posterior colliculus (coronal view); type I fractures may be fixed via a posterolateral approach alone. In type II fractures, the posterior fragment is split into 2 pieces, a posterolateral fragment and a posteromedial fragment involving the medial malleolus; type II fractures may require an additional posteromedial approach to assist in the reduction and fixation of the posteromedial fragment. In type III fractures, a long oblique fracture line divides the fragment into 2 pieces and runs through the anterior portion of the medial malleolus; based on the procedure for type II fractures, a posteromedial approach is always required for the reduction and fixation of medial malleolus fragments. This classification system cannot only describe the severity of posterior pilon fractures but also guide the selection of an appropriate surgical approach and internal fixation strategy. The current classification system is based on CT images, which are required for the diagnosis of posterior pilon fractures $(9,10)$.

Posterior pilon fractures are frequently associated with injury of the lower syndesmosis ligament. This ligament originates from the posterior malleolus, attaches to the posterior portion of the lateral malleolus and is an important structure for maintaining ankle stability $(11,12)$. In addition, posterior pilon fractures may be accompanied by the dislocation of the talus or comminuted lateral (medial) malleolus fractures. Thus, posterior pilon fractures are a fracture pattern between typical posterior malleolus fractures and pilon fractures, and are considered unstable. Thus, it is necessary to select a reliable reduction and fixation technique in clinical treatments. Longer ankle immobilization is required in these cases, and 2 or 3 weeks of cast immobilization is recommended. The patients may start weight-bearing exercise at week 6-12 and walk while bearing full weight at 3 months $(2,6,9)$.

Anatomic reduction is the first principle in the treatment of posterior pilon fractures. Following reduction, the residue step in the articular surface of the distal tibia should not exceed $1 \mathrm{~mm}$. Verhage et al (13) performed a follow-up study in 52 patients with trimalleolar fractures, in the surgery of which patients were placed in the prone position. The posterolateral approach to the ankle provides adequate access to the posterior malleolus, allowing its anatomical reduction and stable fixation. Complications rarely occur when using this approach. In terms of fixation, the majority of clinicians prefer the posterolateral approach and supportive plate fixation, particularly in patients with osteoporosis (14-16). Certain clinicians have opted for cannulated compression screw fixation (from posterior to anterior) and have achieved satisfactory results $(2,6)$. In addition, a plate and screws may be used to fix the posterolateral and posteromedial bone fragments, respectively, depending on the condition of each case (17).

In certain cases, the peroneal tendon is entrapped between the fibula fracture fragment and the posterior fragments of the tibia, and is the major case of reduction failure in this pattern of fracture with posterior dislocation of the talus. Lu et al (18). Reported a rare case of posterior pilon fracture combined with irreducible dislocation and proposed a radiological characteristic, 'tongues of flame sign', which indicates peroneal tendon entrapment between the fibula and the posterior tibial fracture gap. All types of posterior pilon fracture require open reduction. However, the fracture fragment cannot be turned over due to the limited exposure of the posterior tibia and the intact inferior tibiofibular ligament. In addition, only the posterior tibial fracture line may be visualized in the incision. An attempt to turn the fragment may result in greater tissue damage. In the present study, the posterior fracture fragment of the tibia was exposed via the fibula fracture gap. Kirschner wires were used to retract the fibula fracture fragment to expose the posterior edge of the tibia. This method may clearly expose the incarcerated soft tissue and the fracture fragments between the tibia fracture fragments. All incarcerated tissues should be removed. Following the reduction of the fibular fractures, K-wires may be used for temporary fixation rather than using a plate, which may interfere with the obtainment of satisfactory fluoroscopic images during surgery. If additional posteromedial fragments are present, a posteromedial incision is required to assist fixation. Either a plate or screws may be selected for fixation.

There is a risk of damaging the sural nerve or posterior tibial neurovascular bundles when using posterolateral or additional posteromedial incisions, particularly in patients requiring removal of internal fixations. The scars and adhesions in the healed incision may be a challenge in secondary surgeries, and an inexperienced surgeon may be more likely to cause intraoperative damage during internal fixation removal. Post-operative complications include wound infection, restrictions in ankle mobility, hardware irritability and re-displacement of the fracture. The incidence of postoperative complications was reported as $11-47 \%(5,13-15)$. In the present study, postoperative complications occurred in 5 patients $(21.7 \%)$, and 1 patient had a post-operative wound infection; the wound was healed following local treatment. One patient had post-operative numbness around the incision and the dorsum of the foot. The numbness gradually subsided after 4 months of treatment with a neurotrophic agent. A total of 3 patients complained of persistent pain, which improved following the removal of their internal fixations. At the final follow-up in the present study, the functional results were favorable with a mean AOFAS score of 87.3, the anatomical reduction rate was $73.9 \%$, which was similar to the results of other studies $(13,14)$. Furthermore, their ankle mobility recovered well. Based on the AOFAS, the average score for flexion and extension was 6.3 points (maximum of 8 points), and the mean score for inversion and eversion was 5.2 points (maximum of 6 points). A multicenter randomized controlled study including 110 patients with unstable ankle fractures undergoing surgical treatment reported that early (2 weeks) ankle weight-bearing and motion exercise may improve the functional prognosis of the ankle without increasing the incidence of complications (19).

With the popularity of CT equipment in clinical settings, patients who are diagnosed with ankle fracture by X-ray examinations may be confirmed by CT. Therefore, it is not difficult to make a definitive diagnosis of posterior pilon fracture. Anatomical reduction and proper fixation of posterior fracture 
fragments are essential for favorable prognoses. However, conventional ankle fracture reductions, in which lateral malleolus fractures are first reduced to achieve a reduction of the posterior malleolus fracture via inferior tibiofibular ligament retraction, may not restore a smooth articular surface. Therefore, it is important to understand the definitions, classifications and treatment guidelines of posterior fractures to develop a suitable and reasonable treatment plan.

Notably, there were some limitations in the present study. Limitations of the present study included the intrinsic weakness of a retrospective study and lack of powerful statistical data to reveal the advantage of the plate fixation method. In addition, the incidence of the specific type of fracture is low, so sample limitation is inevitable.

In conclusion, posterior pilon fractures are a common pattern of ankle fractures. Achieving good clinical efficacy requires the selection of an appropriate surgical approach and proper post-operative rehabilitation. In the present study, a posterolateral incision approach was employed that was able to clearly expose the operative field without requiring the turning over of the posterior fracture fragment of the tibia. The fibula fracture gap was used to expose the incarcerated tissues between the tibial fracture fragments and to reduce the fractures with minimal trauma. Posterior placement of a plate may be employed to maintain the stability of the anatomical reduction of the fracture and promote early functional rehabilitation. Therefore, this procedure may reduce the complication rate and is helpful in post-operative recovery; thus, it is a safe and effective internal fixation method.

\section{Acknowledgements}

Not applicable.

\section{Funding}

No funding received.

\section{Availability of data and materials}

The datasets used and/or analyzed during the present study are available from the corresponding author on reasonable request.

\section{Authors' contributions}

MG and NL collected clinical samples and wrote the manuscript. YC and WS performed the experiments and statistical analyses. HY designed the present study and revised the manuscript. All authors read and approved the final manuscript.

\section{Ethics approval and consent to participate}

The present study was approved by the Ethics Committee of the First Affiliated Hospital of Soochow University (Suzhou, China). Written informed consent was obtained from all patients.

\section{Patient consent for publication}

Not applicable.

\section{Competing interests}

The authors declare that they have no competing interests.

\section{References}

1. Klammer G, Kadakia AR, Joos DA, Seybold JD and Espinosa N: Posterior pilon fractures: A retrospective case series and proposed classification system. Foot Ankle Int 34: 189-199, 2013.

2. Amorosa LF, Brown GD and Greisberg J: A surgical approach to posterior pilon fractures. J Orthop Trauma 24: 188-193, 2010.

3. Switaj PJ, Weatherford B, Fuchs D, Rosenthal B, Pang E and Kadakia AR: Evaluation of posterior malleolar fractures and the posterior pilon variant in operatively treated ankle fractures. Foot Ankle Int 35: 886-895, 2014.

4. Topliss CJ, Jackson M and Atkins RM: Anatomy of pilon fractures of the distal tibia. J Bone Joint Surg Br 87: 692-697, 2005.

5. Forberger J, Sabandal PV, Dietrich M, Gralla J, Lattmann T and Platz A: Posterolateral approach to the displaced posterior malleolus: functional outcome and local morbidity. Foot Ankle Int 30: 309-314, 2009.

6. Wang L, Shi ZM, Zhang CQ and Zeng BF: Trimalleolar fracture with involvement of the entire posterior plafond. Foot Ankle Int 32: 774-781, 2011.

7. Fonseca LLD, Nunes IG, Nogueira RR, Martins GEV, Mesencio AC and Kobata SI: Reproducibility of the Lauge-Hansen, Danis-Weber, and AO classifications for ankle fractures. Rev Bras Ortop 53: 101-106, 2017.

8. Burwell HN and Charnley AD: The treatment of displaced fractures at the ankle by rigid internal fixation and early joint movement. J Bone Joint Surg Br 47: 634-640, 1965.

9. Li M, Collier RC, Hill BW, Slinkard N and Ly TV: Comparing different surgical techniques for addressing the posterior malleolus in supination external rotation ankle fractures and the need for syndesmotic screw fixation. J Foot Ankle Surg 56: 730-734, 2017.

10. Evers J, Barz L, Wähnert D, Grüneweller N, Raschke MJ and Ochman S: Size matters: The influence of the posterior fragment on patient outcomes in trimalleolar ankle fractures. Injury 46 (Suppl 4): S109-S113, 2015.

11. Golanó P, Vega J, de Leeuw PA, Malagelada F, Manzanares MC, Götzens V and van Dijk CN: Anatomy of the ankle ligaments: A pictorial essay. Knee Surg Sports Traumatol Arthrosc 24: 944-956, 2016.

12. Evers J, Fischer M, Zderic I, Wähnert D, Richards RG, Gueorguiev B, Raschke MJ and Ochman S: The role of a small posterior malleolar fragment in trimalleolar fractures: A biomechanical study. Bone Joint J 100-B: 95-100, 2018.

13. Verhage SM, Boot F, Schipper IB and Hoogendoorn JM: Open reduction and internal fixation of posterior malleolar fractures using the posterolateral approach. Bone Joint J 98-B: 812-817, 2016.

14. Chen DW, Li B, Aubeeluck A, Yang YF, Zhou JQ and Yu GR: Open reduction and internal fixation of posterior pilon fractures with buttress plate. Acta Ortop Bras 22: 48-53, 2014.

15. Wang Y, Wang $\mathrm{J}$ and Luo CF: Modified posteromedial approach for treatment ofposterior pilon variant fracture. BMC Musculoskelet Disord 17: 328, 2016.

16. Abdelgawad AA, Kadous A and Kanlic E: Posterolateral approach for treatment of posterior malleolus fracture of the ankle. J Foot Ankle Surg 50: 607-611, 2011.

17. Mak MF, Stern R and Assal M: Repair of syndesmosis injury in ankle fractures: Current state of the art. EFORT Open Rev 3: 24-29, 2018.

18. Lu J, Maruo Holledge M, Trappel J and Mayank M: A radiological sign (which we are calling the 'tongues of flame' sign) in irreducible trimalleolar fractures of the ankle. Foot Ankle Surg 22: e6-e9, 2016.

19. Dehghan N, McKee MD, Jenkinson RJ, Schemitsch EH, Stas V, Nauth A, Hall JA, Stephen DJ and Kreder HJ: Early weightbearing and range of motion versus non-weightbearing and immobilization after open reduction and internal fixation of unstable ankle fractures: A randomized controlled trial. J Orthop Trauma 30: 345-352, 2016. 